ARTICLE

\title{
Multi-omics prediction of immune-related adverse events during checkpoint immunotherapy
}

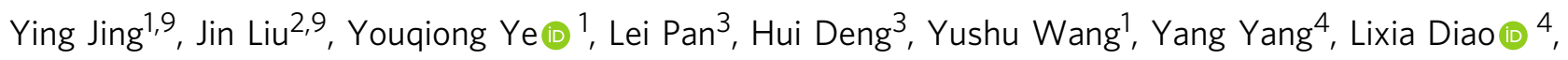

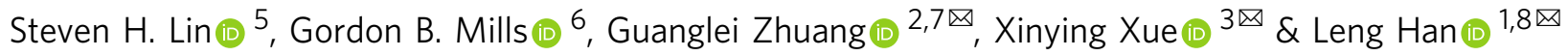

Immune-related adverse events (irAEs), caused by anti-PD-1/PD-L1 antibodies, can lead to fulminant and even fatal consequences and thus require early detection and aggressive management. However, a comprehensive approach to identify biomarkers of irAE is lacking. Here, we utilize a strategy that combines pharmacovigilance data and omics data, and evaluate associations between multi-omics factors and irAE reporting odds ratio across different cancer types. We identify a bivariate regression model of LCP1 and ADPGK that can accurately predict irAE. We further validate LCP1 and ADPGK as biomarkers in an independent patient-level cohort. Our approach provides a method for identifying potential biomarkers of irAE in cancer immunotherapy using both pharmacovigilance data and multiomics data.

\footnotetext{
${ }^{1}$ Department of Biochemistry and Molecular Biology, The University of Texas Health Science Center at Houston McGovern Medical School, Houston, TX 77030, USA. ${ }^{2}$ State Key Laboratory of Oncogenes and Related Genes, Shanghai Cancer Institute, Ren Ji Hospital, School of Medicine, Shanghai Jiao Tong University, 200217 Shanghai, China. ${ }^{3}$ Department of Respiratory and Critical Care, Beijing Shijitan Hospital, Capital Medical University; Peking University Ninth School of Clinical Medicine, 100038 Beijing, China. ${ }^{4}$ Department of Bioinformatics and Computational Biology, The University of Texas MD Anderson Cancer Center, Houston, TX 77030, USA. ${ }^{5}$ Department of Radiation Oncology, Division of Radiation Oncology, The University of Texas MD Anderson Cancer Center, Houston, TX 77030, USA. ${ }^{6}$ Department of Cell, Development, and Cancer Biology, Knight Cancer Institute, Oregon Health and Science University, Portland, OR 97239, USA. ${ }^{7}$ Shanghai Key Laboratory of Gynecologic Oncology, Ren Ji Hospital, School of Medicine, Shanghai Jiao Tong University, 200217 Shanghai, China. ${ }^{8}$ Center for Precision Health, The University of Texas Health Science Center at Houston, Houston, TX 77030, USA

${ }^{9}$ These authors contributed equally: Ying Jing, Jin Liu. ${ }^{凶}$ email: zhuangguanglei@gmail.com; xinyingxue2010@163.com; leng.han@uth.tmc.edu
} 
mmune-related adverse events (irAEs) during antiprogrammed death 1 (PD-1) or anti-programmed death ligand 1 (PD-L1) antibody therapy, resulting from immune activation combined with disturbed immunologic homeostasis, can affect any organ systems and in some cases can be lethal ${ }^{1}$. Pneumonitis, the most common fatal irAE, results in $10 \%$ death rate, and accounts for $35 \%$ of anti-PD-1/PD-L1-related fatalities ${ }^{2}$. Myocarditis, the most lethal irAE, causes $\sim 50 \%$ mortality $^{3}$. Therefore, the predictive biomarkers of irAEs are required to determine the benefit/risk ratio for patients receiving anti-PD-1/ PD-L1 therapy. T-cell receptor (TCR) diversity ${ }^{4}, \mathrm{CD} 8+\mathrm{T}$-cell clonal expansion ${ }^{5}$, and tumor mutational burden $(\mathrm{TMB})^{6}$ have been suggested to potentially predict irAE albeit on the basis of a single factor or conducted in a limited number of cases. Therefore, a comprehensive approach to identify biomarkers of irAE is lacking. In particular, it is challenging to obtain a patient sample cohort with enough sample size, and the traditional approach may require multiple years of multi-center efforts.

In this study, we investigate potential predictors for irAE risk in patients receiving anti-PD-1/PD-L1 therapies across 26 tumor types by integrating real-world pharmacovigilance and molecular omics data. We identify the bivariate linear-regression model of LCP1 and ADPGK that can accurately predict irAE, and validate LCP1 + ADPGK model predictive potential in an independent patient cohort. Our approach provides a method for identifying potential biomarkers of irAE in cancer immunotherapy.

\section{Results}

Analysis of known factors in predicting irAE. To identify potential biomarkers of irAE in anti-PD-1/PD-L1 therapy, we retrieved from the US Food and Drug Administration Adverse Event Reporting System (FAERS) a total of 52,282 adverse events (AEs) from 18,706 patients for 26 different cancer types receiving anti-PD-1/PD-L1 therapy. Among these patients, 3706 (19.8\%) had at least one irAE. We calculated the irAE reporting odds ratio (ROR) by comparing the proportion of reporting irAEs for antiPD-1/PD-L1 agents with the proportion of reporting irAEs for all other drugs in the database ${ }^{7}$. IrAE ROR varied by tumor type and the highest irAE ROR observed for lung adenocarcinoma (LUAD) (3.29, 95\% confidence interval (CI), 2.97-3.65), while the lowest value was observed for uterine carcinosarcoma (UCS) (0.65, 95\% CI, 0.02-4.18) (Fig. 1a and Supplementary Table 1). We collected six factors related to irAEs, including $\mathrm{TMB}^{6}$, T-cell receptor (TCR) diversity ${ }^{8}$, interferon (IFN) a level $^{9}$, tumor necrosis factor (TNF) a level ${ }^{9}$, eosinophils ${ }^{10}$, and neutrophils ${ }^{11}$. Interestingly, these factors are also biomarkers of immune therapy response based on positive associations between the incidence of irAEs and benefit for patients treated with immune checkpoint inhibitors $^{12,13}$. Indeed, we observed a marginally significant correlation between irAE ROR and objective response rates ${ }^{14}$ in antiPD-1/PD-L1 therapy (Rs $=0.44 ; \quad P=0.049 ;$ Supplementary Fig. 1). We further collected 36 factors related to immune therapy response, including $\mathrm{TMB}^{15}$, cytolytic activity ${ }^{16}$, and neoantigen load ${ }^{15}$. We evaluated the association of these factors calculated from molecular data of The Cancer Genome Atlas (TCGA) and irAE risks based on the individual safety reports from FAERS. We identified seven potential predictors, including cytolytic activity (Spearman $R, R s=0.64$; false discover rate $(\mathrm{FDR})=0.01)$, IFN $\gamma$ signature $(R s=0.61, \mathrm{FDR}=0.01), \mathrm{PD}-1$ expression $(R s=0.60$, $\mathrm{FDR}=0.01)$, TCR diversity $(R s=0.59, \mathrm{FDR}=0.01)$, macrophages M1 $(R s=0.55, \mathrm{FDR}=0.03), \mathrm{CD} 8+\mathrm{T}$-cell abundance $(R s$ $=0.50, \quad \mathrm{FDR}=0.05)$, naive $\mathrm{B}$ cells $(R s=0.49, \quad \mathrm{FDR}=0.05)$ (Fig. 1b, Supplementary Fig. 2, and Supplementary Table 2). To identify more powerful predictive models, we combined these seven factors and evaluated the performance of bivariate models by Spearman correlation and goodness of fit by the log-likelihood ratio test ${ }^{17}$. The combination of $\mathrm{CD} 8+\mathrm{T}$-cell abundance with TCR diversity or naive $\mathrm{B}$ cells achieved significantly improved goodness of fit of models compared to using the single factors (Fig. 1c and Supplementary Fig. 3). In particular, the combination of CD8 + T cells and TCR diversity achieved maximum predictive efficacy $\left(R s=0.75\right.$, FDR $\left.=8.24 \times 10^{-4}\right) \quad$ (Fig. 1d and Supplementary Table 3$)$. The correlation coefficient $(R s, 0.75)$ suggested that $56 \%\left(R s^{2}, 0.56\right)$ of observed irAE ROR was explained by this bivariate regression model. We assessed the multicollinearity of these seven factors by the variance inflation factor ${ }^{18,19}$, and observed no multicollinearity for TCR diversity and CD8+ T cells (Supplementary Fig. 4). We also found that TCR diversity and CD8 + T-cell abundance exhibited no significant correlation $(P=$ $0.26)$, suggesting the independent prediction of irAE. We further evaluated the performance of the combinations of other factors with TCR diversity-CD8+ T-cell abundance bivariate model. No trivariate models achieved higher correlation coefficients or increased accuracy (Supplementary Table 4).

Comprehensive identification for potential irAE biomarkers. We further sought to identify additional predictive factors for irAE by conducting a comprehensive screening across mRNA, miRNA, lncRNA and protein expression, and nonsilent gene mutations across 26 cancer types. The majority of the top hits that resulted were gene expressions (Supplementary Table 5), which were highly enriched in immune response processes, including T-cell activation and cell killing (Fig. 2a). This provided further support for the $\mathrm{T}$ cells as the pivotal regulators in irAEs. Of particular interest, the lymphocyte cytosolic protein 1 (LCP1), which is involved in T-cell activation ${ }^{20}$, achieved the highest correlation coefficient $\left(R s=0.82, \mathrm{FDR}=6.69 \times 10^{-3}\right.$, Fig. $\left.2 \mathrm{~b}\right)$. Combinations between any two of the top ten significant irAE correlated genes (Supplementary Fig. 5) suggested that the combination of LCP1 with most of the other genes achieved better predictive performance (Fig. 2c, Supplementary Fig. 6, and Supplementary Table 6). Among these, adding the adenosine diphosphate dependent glucokinase $(A D P G K)$, which is mediating metabolic shift during T-cell activation ${ }^{21}$, to $L C P 1$ led to a linear-regression model with the best accuracy among all the bivariate models $\left(R s=0.91, \mathrm{FDR}=7.94 \times 10^{-9}\right.$, Fig. $2 \mathrm{~d}$ and Supplementary Table 6). We evaluated the multicollinearity of these top ten genes by the variance inflation factor ${ }^{18,19}$ and observed no multicollinearity for LCP1 and ADPGK (Supplementary Fig. 7). Combinations of the third gene did not improve the predictive value of the $L C P 1$ and $A D P G K$ bivariate model (Supplementary Table 7). We further screened the combination of significant factors and genes to identify more powerful combinations, and did not discover models have better performance (Supplementary Figs. 8 and 9, and Supplementary Table 8). Considering the straightforward estimation of $L C P 1$ and $A D P G K$, this combination maybe easier to translate into clinical practice. As far as we know, no study reported that $L C P 1$ and/or $A D P G K$ is associated with immunotherapy response yet. We further performed correlation analysis between objective response rate $\mathrm{e}^{14}$ and $L C P 1 / A D P G K$, and observed no significant associations (Supplementary Fig. 10), suggesting limited confounding effect from efficacy, at least for $L C P 1$ and $A D P G K$.

Validation of LCP1 and ADPGK as irAE biomarkers. To validate the predictive power of $L C P 1$ and $A D P G K$, we further collected a validation cohort of 28 cancer patients receiving antiPD-1/PD-L1 inhibitors with both high-quality formalin-fixed paraffin-embedded (FFPE) pre-treatment tumor tissues and clinicopathological information (Supplementary Table 9 and 
a

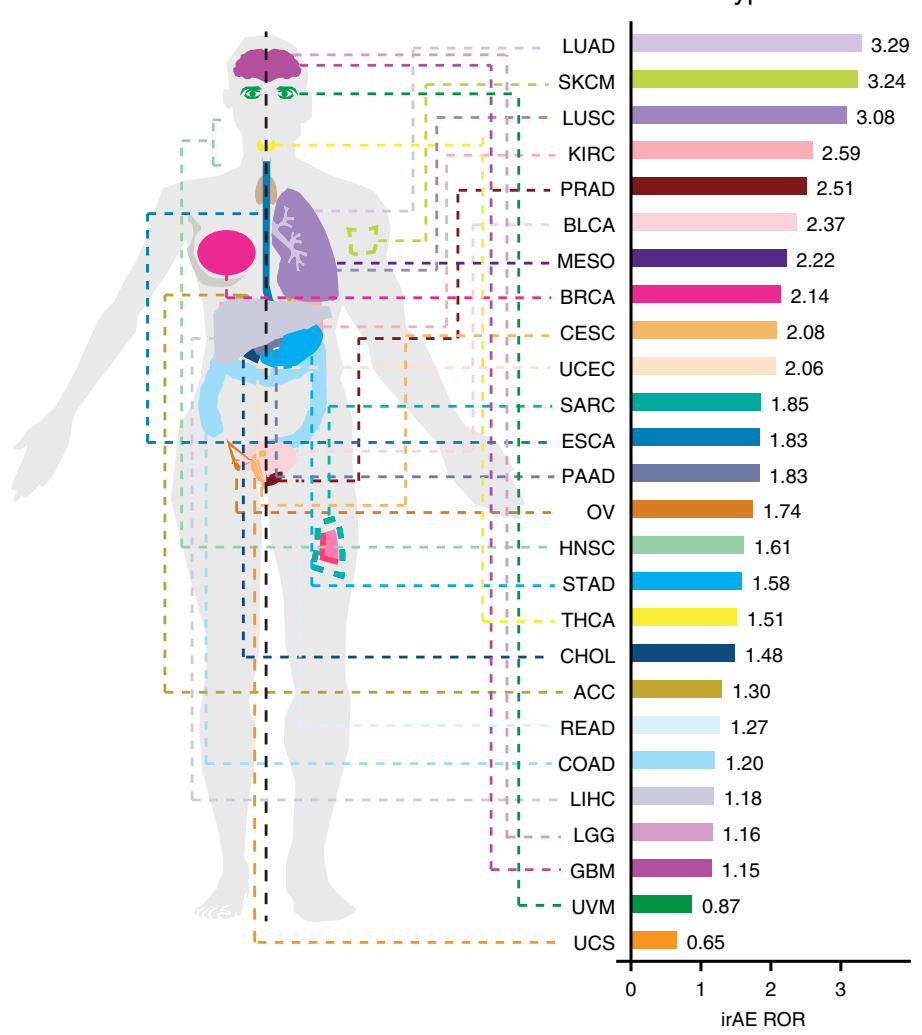

b

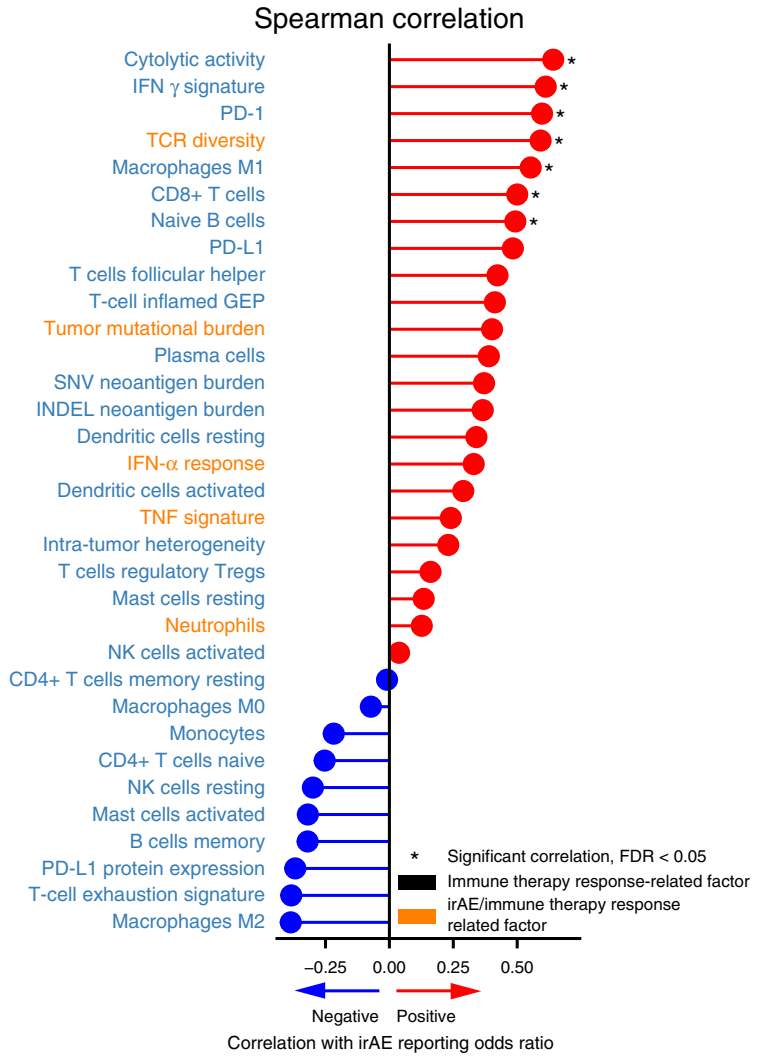

C

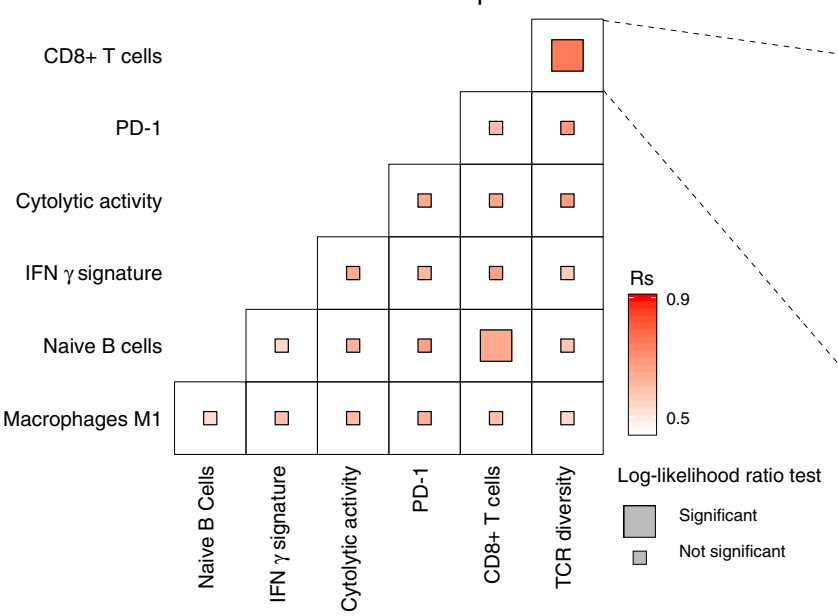

TCR diversity + CD8+ T cells

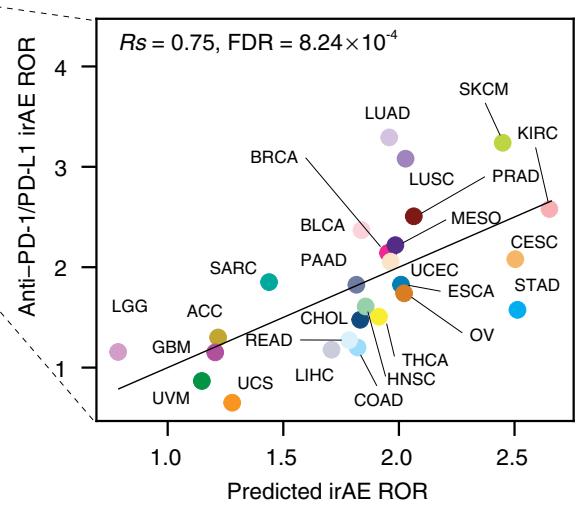

Cancer types

ACC LUSC

BLCA MESO

BRCA OV

- CESC PAAD

- ChOl PRAD

coad READ

- esca SARc

- GBM SKCM

HNSC STAD

- KIRC THCA

- LGG UCEC

LIHC UVM

- LUAD UCS

Fig. 1 Evaluation of the association between irAE and related factors. a Anatomic sites of cancer types (left panel), and irAE ROR across 26 cancer types (right panel). b Spearman correlation between irAE ROR and 36 factors for positive correlation (red lollipop) and negative correlation (blue lollipop). * indicates significant correlation (FDR $<0.05$ ); cytolytic activity FDR $=0.01$, Interferon (IFN) $\gamma$ signature FDR $=0.01, \mathrm{PD}-1 \mathrm{FDR}=0.01$, $\mathrm{T}$-cell receptor (TCR) diversity FDR $=0.01$, macrophages $M 1 F D R=0.03, C D 8+T$ cells FDR $=0.05$, naive $B$ cells FDR $=0.05$; irAE and immune therapy response-related factors are marked in orange. c Comparison of performance of bivariate models in predicting irAE for all combinations of six significantly correlated variables. Spearman R (Rs) was calculated between predicted and observed irAE ROR. The shade of the square indicates the Rs, and the size indicates the significance of the log-likelihood ratio test. $\mathbf{d}$ Combined effect of TCR diversity and CD8+ T-cell bivariate model (Spearman correlation, $R s=0.75$, FDR $=$ $8.24 \times 10^{-4}$ ). The equation of the bivariate model is $0.31 \times \mathrm{TCR}$ diversity $+8.87 \times \mathrm{CD} 8+\mathrm{T}$ cells +0.27 . irAE immune-related adverse events, ROR reporting odds ratio, FDR false discovery rate, LUAD lung adenocarcinoma, SKCM skin cutaneous melanoma, LUSC lung squamous cell carcinoma, KIRC kidney renal clear cell carcinoma, PRAD prostate adenocarcinoma, BLCA bladder urothelial carcinoma, MESO mesothelioma, BRCA breast invasive carcinoma, CESC cervical squamous cell carcinoma and endocervical adenocarcinoma, UCEC uterine corpus endometrial carcinoma, SARC sarcoma, ESCA esophageal carcinoma, PAAD pancreatic adenocarcinoma, OV ovarian serous cystadenocarcinoma, HNSC head and neck squamous cell carcinoma, STAD stomach adenocarcinoma, THCA thyroid carcinoma, CHOL cholangiocarcinoma, ACC adrenocortical carcinoma, READ rectum adenocarcinoma, COAD colon adenocarcinoma, LIHC liver hepatocellular carcinoma, LGG brain lower-grade glioma, GBM glioblastoma multiforme, UVM uveal melanoma, UCS uterine carcinosarcoma. 
a

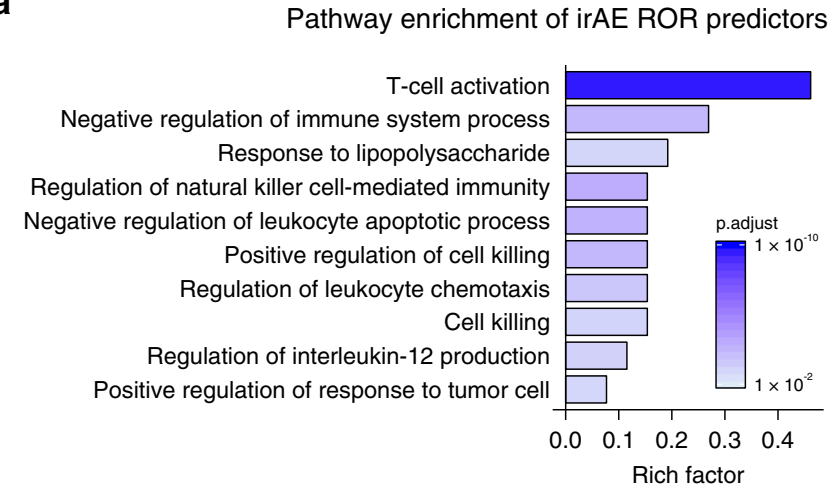

b

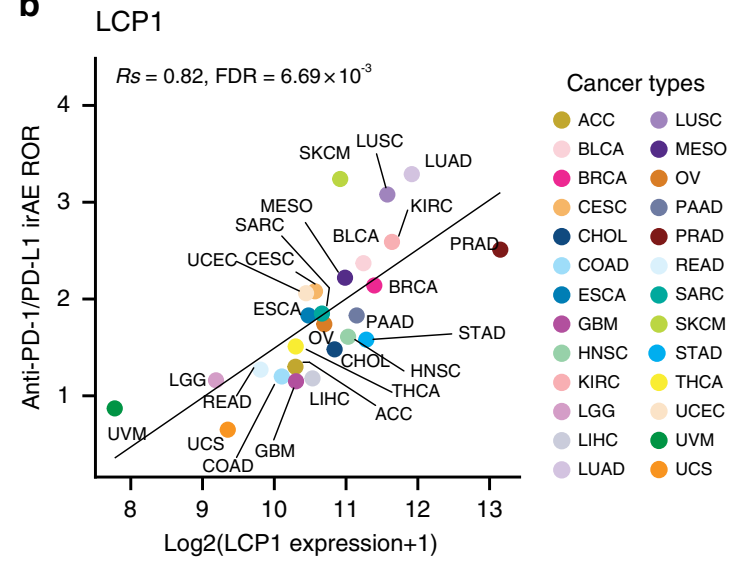

c

Bivariate model comparison

d

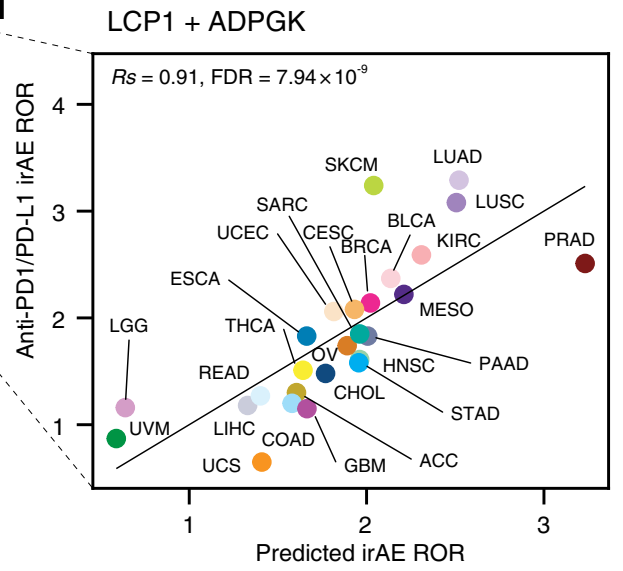

Log-likelihood ratio test

Significant

$\square$ Not significant

Predicted irAE ROR

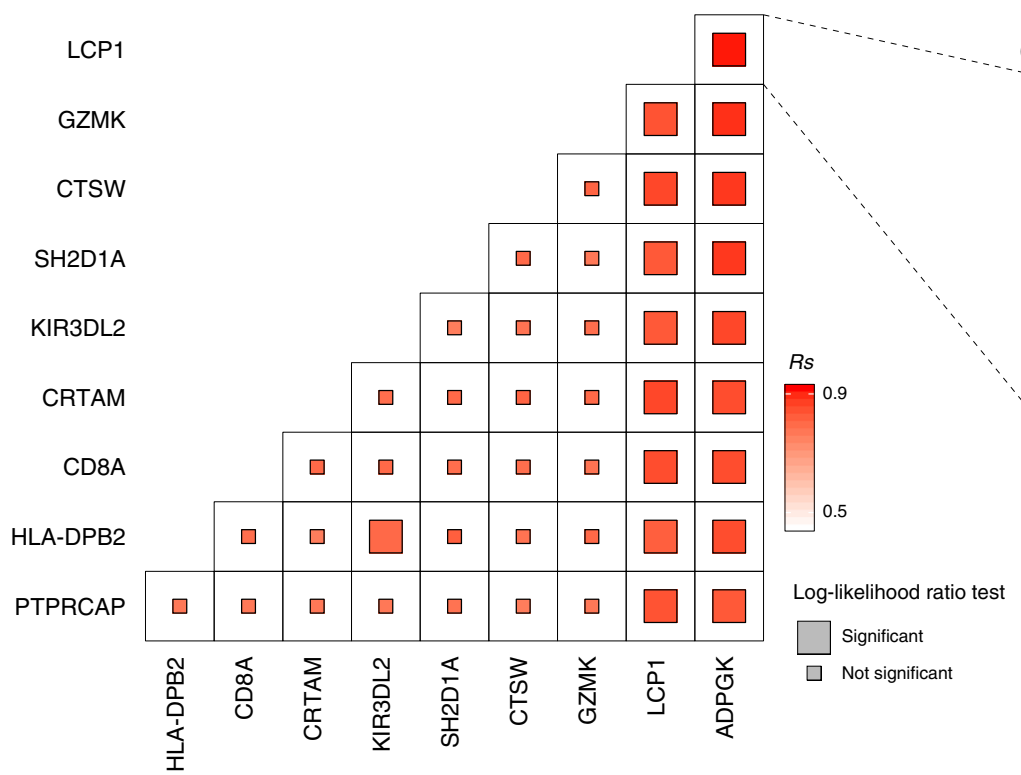

Fig. 2 Comprehensive identification of potential irAE predictors. a Pathway enrichment of the top ten genes significantly correlated with irAE ROR across multiple cancer types. $\mathbf{b}$ Spearman correlation between LCP1 expression and irAE ROR. c Comparison of performance of bivariate models in predicting irAE for all combinations of the top ten irAE ROR significantly correlated genes. Spearman correlation (Rs) was calculated between the predicted and observed irAE ROR. The shade of the square indicates the Rs, and the size indicates the significance of the log-likelihood ratio test. $\mathbf{d}$ Combined effect of LCP1 and ADPGK bivariate model (Spearman correlation, $R s=0.91, F D R=7.94 \times 10^{-9}$ ). The equation of the bivariate regression model is $0.37 \times L C P 1+0.70 \times$ ADPGK - 9.10.

Supplementary Fig. 11). The median age of the patients was 56 years (range, 37-82 years), with $22(78.6 \%)$ male patients and 6 (21.4\%) female patients. In all, 26 of $28(92.9 \%)$ patients were diagnosed as lung cancer. The expression level of LCP1 and ADPGK were assessed by immunohistochemistry in our validation cohort. LCP1 and ADPGK have stronger staining in the irAE group (Fig. 3a). We quantified the immunostaining signals for the protein expression of LCP1 and ADPGK using the Aperio ImageScope software v14.3 with Positive Pixel Count v9 (PPCv9) algorithm. Consistently, LCP1 $(P$ value $=0.008)$ and ADPGK $(P$ value $=0.010)$ were higher in patients with irAEs when compared with patients without irAEs (Fig. 3b). The geometric mean of LCP1 and ADPGK was also higher in pre-treatment tumor samples of patients with irAEs $(P$ value $=0.005$, Fig. $3 c)$. The area under the receiver-operating characteristic curve (AUC) of LCP1 and ADPGK to predict irAE was 0.78 and 0.78 , while the combination of LCP1 and ADPGK had a better AUC value as 0.80 (Fig. 3d). Furthermore, LCP1, ADPGK, and LCP1+ADPGK successfully predicted pneumonitis in 26 lung cancer patients with AUC as 0.74, 0.76, and 0.77, respectively (Supplementary
Fig. 12), suggesting the potential utility of LCP1 and ADPGK in predicting a specific type of irAE in a specific cancer. We further tested the CD8 predictive potential in our validation cohort. We did not observe significantly increased CD8 IHC staining signals in pre-treatment tumors in the irAE group. In addition, we found the AUC value of CD8 is not comparable to LCP1 and ADPGK (Supplementary Fig. 13), suggesting that the confounding effect of CD8 in irAE prediction is limited. Taken together, this independent patient-level cohort validated the predictive power of LCP1 and ADPGK for irAEs in cancer patients receiving antiPD-1/PD-L1 inhibitors.

\section{Discussion}

In this study, we systematically investigated potential predictors for irAE risk in patients receiving anti-PD-1/PD-L1 therapies across 26 tumor types by integrating real-world and molecular data. We identified seven potential predictors, and the combination of CD8 $+\mathrm{T}$ cells and TCR diversity achieved higher accuracy of prediction of irAE and decreased the unexplained 
a
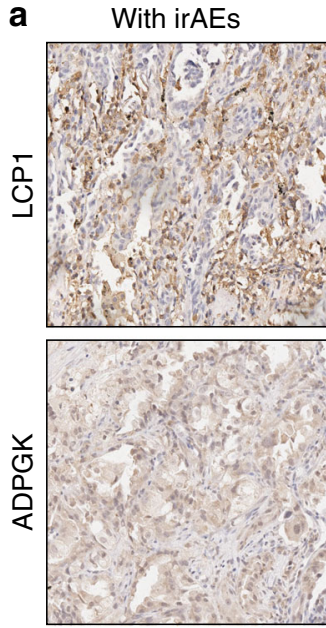

Without irAEs
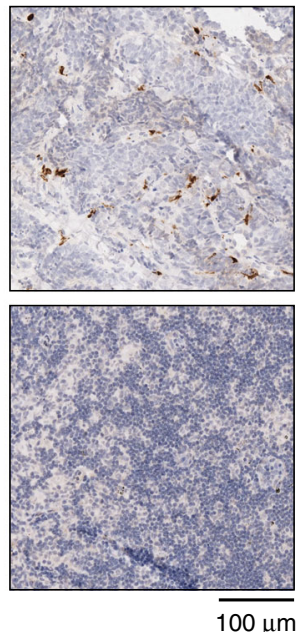

b

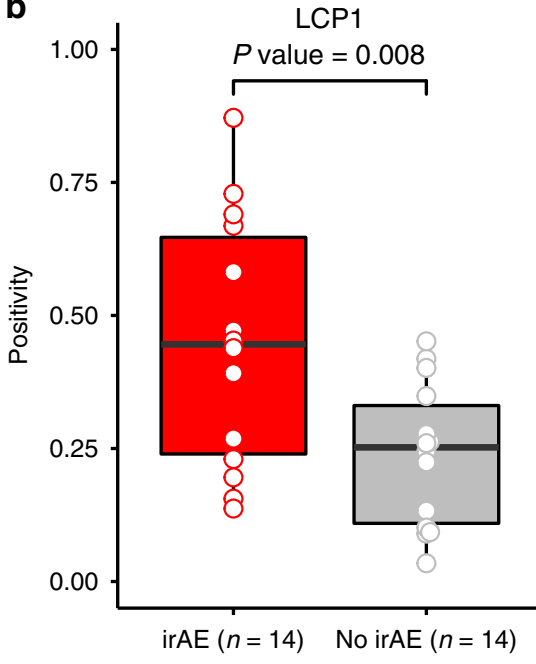

ADPGK

$P$ value $=0.010$
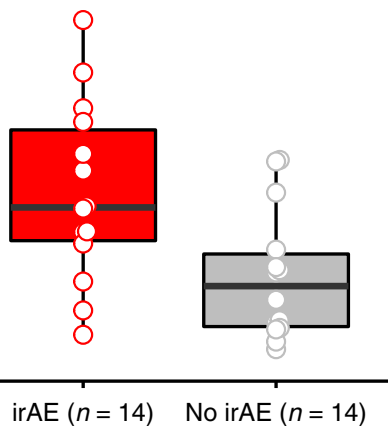

c

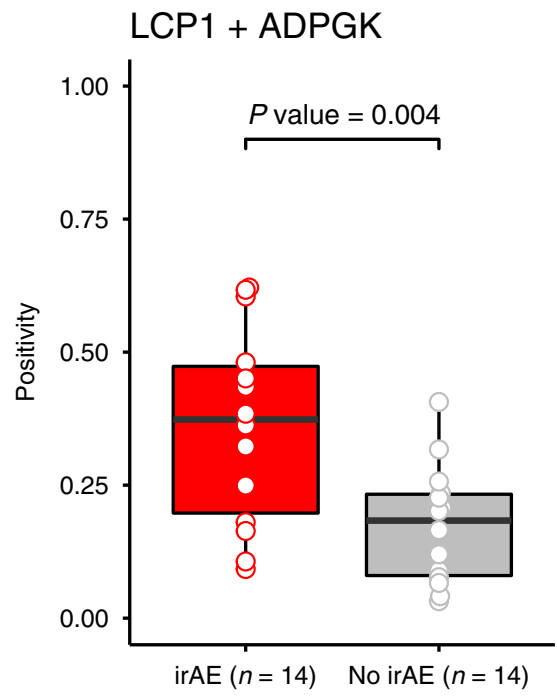

d

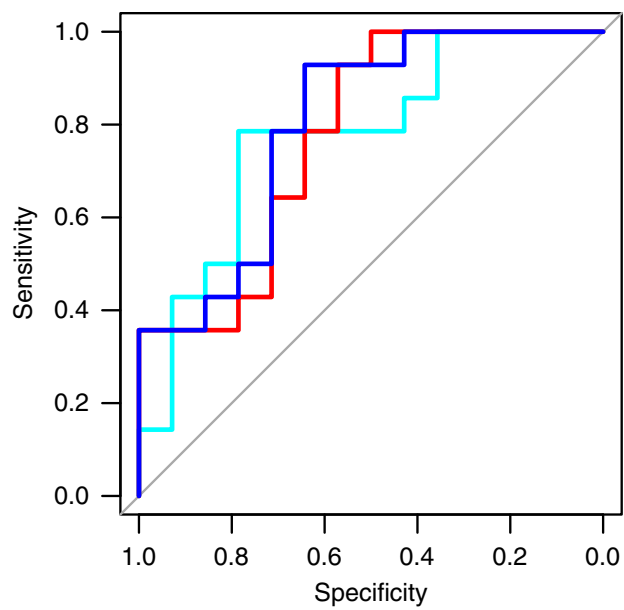

LCP1 + ADPGK: AUC: 0.80

LCP1 AUC: 0.78

ADPGK AUC: 0.78

Fig. 3 Validation of the predictive power of LCP1 and ADPGK in a patient cohort. a Representative images of patients with irAEs and without irAEs through immunohistochemical (IHC) staining with antibodies against ADPGK and LCP1. Image size: $379 \times 379 \mu \mathrm{m}^{2}$; scale bar: $100 \mu \mathrm{m}$. b Quantification of LCP1 and ADPGK IHC staining signals. Comparison between patients with irAE or without irAE is conducted by unpaired two-sided Student's $t$ test. c Geometric mean of LCP1 and ADPGK staining signals. A comparison between patients with irAE and without irAE is conducted by unpaired two-sided Student's $t$ test. d ROC curve of the LCP1, ADPGK, LCP1 + ADPGK in the validation cohort $(n=28)$. The boxes indicate the median \pm 1 quartile, with whiskers extending from the hinge to the smallest or largest value within 1.5 interquartile range from the box boundaries. ROC receiver-operating characteristic, AUC area under the receiver-operating characteristic curve.

variance from $0.59\left(1-0.64^{2}\right)$ to $0.44\left(1-0.75^{2}\right)$. Considering that the unexplained variance was still 0.44 , we further performed a large-scale comprehensive screening to identify better performing irAE ROR predictors. We identified potential irAE predictors that are enriched in the function of T-cell activation. The linearregression model by the combination of $L C P 1$ and $A D P G K$, two genes related to T-cell activation ${ }^{20,21}$, further decreased the unexplained variance from $0.44\left(1-0.75^{2}\right)$ to $0.17\left(1-0.91^{2}\right)$. Our results align well with the emergence of evidence of the involvement of T-cell activation in irAEs. Furthermore, the AUC value of LCP1 and ADPGK in our patient-level validation cohort achieved 0.8 , suggesting that the combination of LCP1 and AGDPGK holds promise as biomarkers for irAEs. Meanwhile, our proof-of-concept study set up an analytic framework to explore irAE biomarkers and propose possible underpinning key factors, which might have important implications for the management of patients with immunotherapy. Our analysis enables the study of promising signals of immune-related toxicities in large sample cohorts, while collecting both molecular data and irAE information of thousands of patients with immunotherapy requires the collaboration of multicenter with several years' efforts. Future work is necessary to further study the predictive performance of LCP1 and ADPGK in larger anti-PD-1/ PD-L1 patient cohorts. We envision that LCP1 and ADPGK might enable a pre-risk-check of patients before receiving antiPD-1/PD-L1 agents with further study.

Nevertheless, our study has a limitation with a limited sample size. Ideally, it will be promising to obtain a large number of patient samples with or without irAEs, and then perform the multi-omics data to identify biomarkers. However, it is challenging to obtain a patient-sample cohort with enough sample size because the percentage of clearly defined of a certain type of irAE, 
e.g., pneumonitis, is $<5 \%^{22,23}$. It may require multiple years of multicenter efforts. Therefore, we utilize an alternative strategy to combine the power of real-world data and omics data. A similar strategy is also utilized to identify the potential biomarkers ${ }^{6,14,24}$, suggesting this strategy is robust and powerful, especially in the absence of a large number of patient samples. Further studies with a larger sample size are necessary to comprehensively identify irAE.

\section{Methods \\ Data analysis of individual safety report from FAERS. We retrieved individual safety reports from FAERS [https://fis.fda.gov/sense/app/d10be6bb-494e-4cd2- 82e4-0135608ddc13/sheet/7a47a261-d58b-4203-a8aa-6d3021737452/state/ analysis] from July 1, 2014 to June 30, 2019. We collected only AE reports from anti-PD-1 agents (nivolumab, pembrolizumab, cemiplimab) and anti-PD-L1 (atezolizumab, avelumab, durvalumab) suspected of causing AEs. We excluded those cases also treated with anti-CTLA-4 agents (ipilimumab, tremelimumab). We used the $\mathrm{AE}$ terms in peer-reviewed irAE management guidelines ${ }^{23}$ to define irAEs. We performed disproportionality analysis ${ }^{7}$ to assess the risk of irAEs via calcu- lating the ROR by using the full database as the comparator. Patients were cate- gorized to irAE group when they have one type of irAEs ${ }^{23}$.}

Data analysis from TCGA and independent datasets. Molecular data, including mRNA expression, miRNA expression, protein expression, and somatic mutations across 26 cancer types were downloaded from TCGA data portal [https://portal. gdc.cancer.gov/]. TCR diversity, neoantigen load, estimated immune cell abundance, and intratumor heterogeneity were downloaded from the GDC PanImmune Data Portal [https://gdc.cancer.gov/about-data/publications/panimmune] ${ }^{25}$. TMB was calculated by the number of nonsilent somatic mutations per sample ${ }^{26}$. We used GSVA R package ${ }^{27}$ v1.3 to compute the T-cell-inflamed gene expression profiling (GEP) level ${ }^{28}$ in each sample based on the T-cell-inflamed GEP signature from Ayers et al. ${ }^{29}$. Cytolytic activity was calculated as the geometric mean of the gene expression of two cytolytic markers (GZMA and PRF1) ${ }^{16}$. IFN $\gamma$ signature was obtained from Ayers et al. ${ }^{29}$.

Identification of biomarkers by combining omics data and real-world data. In our analysis, the number of cancer types is far less than variables (26 cancer types with $>50,000$ variables, including $~ 20,000$ mRNA expression, $\sim 12,000$ noncoding RNA expression, $\sim 18,000$ gene mutations, $\sim 200$ protein expression, and $\sim 2400$ miRNA expression), which may result in an inflated type-I-error and subsequently introduce more false positives ${ }^{30-35}$ if employ other advanced algorithms, e.g., Lasso, Elastic net, and Ridge. Therefore, we adopted an approach as described in a previous study ${ }^{17}$ that they evaluated the correlation between single variables and response rate, and then added variables to obtain bivariate models to achieve better performance. The median values of each factor were calculated for each cancer type. The anatomic illustration was generated by R package gganatogram ${ }^{36,37} \mathrm{vl} .1$. We performed leave-one-out cross-validation in predicting irAE ROR from bivariate and trivariate linear-regression models using the $\mathrm{R}$ package caret v6.0. The predictive performance was estimated using the Spearman rank correlation coefficient $(R s)$ and unexplained variance $\left(1-R s^{2}\right)$. The goodness of fit of the models was compared by the log-likelihood ratio test using the $\mathrm{R}$ package lmtest v0.9. For the bivariate model fitness comparison, the log-likelihood ratio test was performed between two-factor models and the single-factor model with the highest Rs. For the trivariate model fitness comparison, the log-likelihood ratio test was performed between the trivariate model and the bivariate model. We used variance inflation factor to assess multicollinearity by vif function of the car $\mathrm{R}$ package v3.0. Pathway enrichment was conducted using the R package clusterprofiler $^{38} \mathrm{v} 3.14$. The calculation of the ROC curve was completed by pROC R package v1.16. Multiple comparisons were Benjamini-Hochberg adjusted by $\mathrm{p}$. adjust function of the base $\mathrm{R}$ language, version 3.5.0. Statistical significance was defined as two-sided $P<0.05$ and/or FDR $<0.05$.

Immunohistochemistry in our patient cohort. The study was conducted in accordance with ethical guidelines of U.S. Common Rule, and was approved by the Ethics Committee of Beijing Shijitan Hospital. Written informed consent was obtained from all patients. We performed a retrospective review of cancer patients with lung cancer, gastrointestinal (GI) cancer, genitourinary (GU) cancer, or other cancers receiving anti-PD-1/PD-L1 treatment from 2017 to 2019 in Beijing Shijitan Hospital. To identify high-confidence irAE in patients, we used relatively stringent criteria: (1) we only include patients with CT confirmed pneumonitis; (2) we only include the pneumonitis that requires and responds to steroids, immunosuppressants, or endocrine therapies; (3) two investigators independently determine the pneumonitis as immunologic etiology. Patients with incomplete demographic and follow-up information, receiving anti-PD-1/PD-L1 in other hospitals, or a history of anti-CTLA-4 therapy were excluded. Next, we kept patients who have archived high-quality pre-treatment FFPE tumor samples before receiving anti-PD1/PD-L1 and any other treatment. In addition, the tumor tissue should contain
$<30 \%$ necrosis. We collected a comparable number of anti-PD-1/PD-L1-treated cancer patients without any overserved irAEs with matched cancer types, stage, age, sex, and therapy of irAE group (Supplementary Fig. 11). Detailed clinical characteristics of the patient cohort were described in Supplementary Table 9. Immunohistochemistry (IHC) was performed on 5 - $\mu$ m-thick FFPE tumor tissue sections. Slides stained with primary antibodies against LCP1 (1:200, Cell Signaling Technology \#3588), ADPGK (1:900, Novus Biologicals \#NBP1-91653), and CD8 (working solution, MXB Biotechnology \#RMA-0514). Slides were then washed and incubated with horseradish peroxidase-conjugated secondary antibodies (1:200, NeoBioscience, \# ANR02-1). Immunoperoxidase staining was developed using the DAB system according to the manufacturer's instructions (Dako). Slides were counterstained with hematoxylin, dehydrated, and coverslipped using a mounting solution. Whole slides were scanned with an Aperio ScanScope system (Leica Biosystems) and quantified using the Aperio ImageScope software v14.3 with Positive Pixel Count v9 (PPCv9) algorithm. Areas of necrosis or artifacts in the slides were ignored. IHC signals were enumerated in seven random $20 \times$ fields, and averaged signals were used for each slide.

Reporting summary. Further information on research design is available in the Nature Research Reporting Summary linked to this article.

\section{Data availability}

Individual safety records were downloaded from FAERS Public Dashboard [https://fis. fda.gov/sense/app/d10be6bb-494e-4cd2-82e4-0135608ddc13/sheet/7a47a261-d58b-4203a8aa-6d3021737452/state/analysis]. The TCGA data were downloaded from TCGA data portal [https://portal.gdc.cancer.gov/] and GDC PanImmune Data Portal [https://gdc. cancer.gov/about-data/publications/panimmune]. All the remaining data are available within the Article, Supplementary Information files, or available from the author upon reasonable request. Source data are provided with this paper.

Received: 13 March 2020; Accepted: 8 September 2020; Published online: 02 October 2020

\section{References}

1. Postow, M. A., Sidlow, R. \& Hellmann, M. D. Immune-related adverse event associated with immune checkpoint blockade. N. Engl. J. Med. 378, 158-168 (2018).

2. Wang, D. Y. et al. Fatal toxic effects associated with immune checkpoint inhibitors: a systematic review and meta-analysis. JAMA Oncol. 4, 1721-1728 (2018).

3. Salem, J. E. et al. Cardiovascular toxicities associated with immune checkpoint inhibitors: an observational, retrospective, pharmacovigilance study. Lancet Oncol. 19, 1579-1589 (2018).

4. Johnson, D. B. et al. Fulminant myocarditis with combination immune checkpoint blockade. N. Engl. J. Med. 375, 1749-1755 (2016).

5. Subudhi, S. K. et al. Clonal expansion of CD8 T cells in the systemic circulation precedes development of ipilimumab-induced toxicities. Proc. Natl Acad. Sci. USA 113, 11919-11924 (2016).

6. Bomze, D., Hasan Ali, O., Bate, A. \& Flatz, L. Association between immunerelated adverse events during anti-PD-1 therapy and tumor mutational burden. JAMA Oncol. 5, 1633-1635 (2019).

7. Bate, A. \& BA, S. J. E. Quantitative signal detection using spontaneous ADR reporting. Pharmacoepidemiol. Drug Saf. 18, 427-436 (2009).

8. Johnson, D. B. et al. A case report of clonal EBV-like memory CD4+ T cell activation in fatal checkpoint inhibitor-induced encephalitis. Nat. Med. 25, 1243-1250 (2019)

9. Head, L. et al. Biomarkers to predict immune-related adverse events with checkpoint inhibitors. J. Clin. Oncol. 37, 131-131 (2019).

10. Diehl, A., Yarchoan, M., Hopkins, A., Jaffee, E. \& Grossman, S. A. Relationships between lymphocyte counts and treatmentrelated toxicities and clinical responses in patients with solid tumors treated with PD-1 checkpoint inhibitors. Oncotarget 8, 114268-114280 (2017).

11. Fujisawa, Y. et al. Fluctuations in routine blood count might signal severe immune-related adverse events in melanoma patients treated with nivolumab. J. Dermatol. Sci. 88, 225-231 (2017).

12. Sanlorenzo, M. et al. Pembrolizumab cutaneous adverse events and their association with disease progression. JAMA Dermatol. 151, 1206-1212 (2015).

13. Haratani, K. et al. Association of immune-related adverse events with nivolumab efficacy in non-small cell lung cancer. JAMA Oncol. 4, 374-378 (2018).

14. Yarchoan, M., Hopkins, A. \& Jaffee, E. M. Tumor mutational burden and response rate to PD-1 inhibition. N. Engl. J. Med. 377, 2500-2501 (2017).

15. Havel, J. J., Chowell, D. \& Chan, T. A. The evolving landscape of biomarkers for checkpoint inhibitor immunotherapy. Nat. Rev. Cancer 19, 133-150 (2019). 
16. Rooney, M. S., Shukla, S. A., Wu, C. J., Getz, G. \& Hacohen, N. Molecular and genetic properties of tumors associated with local immune cytolytic activity. Cell 160, 48-61 (2015).

17. Lee, J. S. \& Ruppin, E. Multiomics prediction of response rates to therapies to inhibit programmed cell death 1 and programmed cell death 1 ligand 1. JAMA Oncol. 5, 1614-1618 (2019).

18. Oshima, Y., Tanimoto, T., Yuji, K. \& Tojo, A. EGFR-TKI-associated interstitial pneumonitis in nivolumab-treated patients with non-small cell lung cancer. JAMA Oncol. 4, 1112-1115 (2018).

19. Hayashi, T. et al. Visceral adiposity is an independent predictor of incident hypertension in Japanese Americans. Ann. Intern. Med. 140, 992-1000 (2004).

20. Wabnitz, G. H. et al. Costimulation induced phosphorylation of L-plastin facilitates surface transport of the T cell activation molecules CD69 and CD25. Eur. J. Immunol. 37, 649-662 (2007).

21. Kamiński, M. M. et al. T cell activation is driven by an ADP-dependent glucokinase linking enhanced glycolysis with mitochondrial reactive oxygen species generation. Cell Rep. 2, 1300-1315 (2012).

22. Kennedy, L. B. \& Salama, A. K. S. A review of cancer immunotherapy toxicity. CA Cancer J. Clin. https://doi.org/10.3322/caac.21596 (2020).

23. Martins, F. et al. Adverse effects of immune-checkpoint inhibitors: epidemiology, management and surveillance. Nat. Rev. Clin. Oncol. 16, 563-580 (2019).

24. Yarchoan, M. et al. PD-L1 expression and tumor mutational burden are independent biomarkers in most cancers. JCI Insight 4, e126908 (2019).

25. Thorsson, V. et al. The immune landscape of cancer. Immunity 48, 812-830. e14 (2018)

26. Hellmann, M. D. et al. Tumor mutational burden and efficacy of nivolumab monotherapy and in combination with ipilimumab in small-cell lung cancer. Cancer Cell 33, 853-861.e4 (2018).

27. Hänzelmann, S., Castelo, R. \& Guinney, J. GSVA: Gene set variation analysis for microarray and RNA-Seq data. BMC Bioinformatics 14, 7 (2013).

28. Ye, Y. et al. Sex-associated molecular differences for cancer immunotherapy. Nat. Commun. 11, 1779 (2020).

29. Ayers, M. et al. IFN- $\gamma$-related mRNA profile predicts clinical response to PD-1 blockade find the latest version: IFN- $\gamma$-related mRNA profile predicts clinical response to PD-1 blockade. J. Clin. Investig. 127, 2930-2940 (2017).

30. Kwon, S., Oh, S. \& Lee, Y. The use of random-effect models for highdimensional variable selection problems. Comput. Stat. Data Anal. 103, 401-412 (2016).

31. Kirpich, A. et al. Variable selection in omics data: a practical evaluation of small sample sizes. PLoS ONE 13, 1-19 (2018).

32. Vasquez, M. M. et al. Least absolute shrinkage and selection operator type methods for the identification of serum biomarkers of overweight and obesity: simulation and application. BMC Med. Res. Methodol. 16, 1-19 (2016).

33. Zou, H. \& Hastie, T. Regularization and variable selection via the elastic net. J. R. Stat. Soc. Ser. B Stat. Methodol. 67, 301-320 (2005).

34. Zhang, C. H. \& Huang, J. The sparsity and bias of the lasso selection in highdimensional linear regression. Ann. Stat. 36, 1567-1594 (2008).

35. Wu, C. \& Ma, S. A selective review of robust variable selection with applications in bioinformatics. Brief. Bioinform. 16, 873-883 (2014).

36. Maag, J. L. V. gganatogram: an R package for modular visualisation of anatograms and tissues based on ggplot2. F1000Research 7, 1576 (2018).

37. Petryszak, R. et al. Expression Atlas update-an integrated database of gene and protein expression in humans, animals and plants. Nucleic Acids Res. 44, D746-D752 (2016).
38. Yu, G., Wang, L. G., Han, Y. \& He, Q. Y. ClusterProfiler: an R package for comparing biological themes among gene clusters. Omi. A J. Integr. Biol. 16, 284-287 (2012)

\section{Acknowledgements}

This work was supported by the Cancer Prevention and Research Institute of Texas (grant nos. RR150085 and RP190570) to CPRIT Scholar in Cancer Research (L.H.). We thank LeeAnn Chastain for editorial assistance.

\section{Author contributions}

L.H. conceived and supervised the project. Y.J., G.Z., X.X., and L.H. designed and performed the research. Y.J., J.L., Y.Y., Y.W., and L.D. performed data analysis. J.L., L.P., H.D., G.Z., and X.X. collected the validation cohort and performed the IHC staining. Y.J., S.H.L., G.B.M., and L.H. interpreted the results. Y.J., G.B.M., G.Z., and L.H. wrote the paper.

\section{Competing interests}

G.B.M. has sponsored research support from AstraZeneca, Critical Outcomes Technology, Karus, Illumina, Immunomet, Nanostring, Tarveda, and Immunomet, and is on the Scientific Advisory Board for AstraZeneca, Critical Outcomes Technology, ImmunoMet, Ionis, Nuevolution, Symphogen, and Tarveda. The remaining authors declare no competing interests.

\section{Additional information}

Supplementary information is available for this paper at https://doi.org/10.1038/s41467020-18742-9.

Correspondence and requests for materials should be addressed to G.Z., X.X. or L.H.

Peer review information Nature Communications thanks the anonymous reviewers for their contribution to the peer review of this work. Peer reviewer reports are available.

Reprints and permission information is available at http://www.nature.com/reprints

Publisher's note Springer Nature remains neutral with regard to jurisdictional claims in published maps and institutional affiliations.

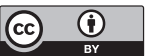

Open Access This article is licensed under a Creative Commons Attribution 4.0 International License, which permits use, sharing, adaptation, distribution and reproduction in any medium or format, as long as you give appropriate credit to the original author(s) and the source, provide a link to the Creative Commons license, and indicate if changes were made. The images or other third party material in this article are included in the article's Creative Commons license, unless indicated otherwise in a credit line to the material. If material is not included in the article's Creative Commons license and your intended use is not permitted by statutory regulation or exceeds the permitted use, you will need to obtain permission directly from the copyright holder. To view a copy of this license, visit http://creativecommons.org/ licenses/by/4.0/.

(C) The Author(s) 2020 